\title{
Contribution and Additional Impact of Imaging to the SPAN-100 Score
}

\author{
P. Krishnan, G. Saposnik, B. Ovbiagele, L. Zhang, S. Symons, and R. Aviv
}

\begin{abstract}
BACKGROUND AND PURPOSE: Stroke Prognostication by Using Age and NIHSS score (SPAN-100 index) facilitates stroke outcomes. We assessed imaging markers associated with the SPAN-100 index and their additional impact on outcome determination.
\end{abstract}

MATERIALS AND METHODS: Of 273 consecutive patients with acute ischemic stroke ( $<4.5$ hours), 55 were characterized as SPAN-100-positive (age +NIHSS score $\geq 100$ ). A comprehensive imaging review evaluated differences, using the presence of the hyperattenuated vessel sign, ASPECTS, clot burden score, collateral score, CBV, CBF, and MTT. The primary outcome assessed was favorable outcome (mRS $\leq 2$ ). Secondary outcomes included recanalization, lack of neurologic improvement, and hemorrhagic transformation. Uni- and multivariate analyses assessed factors associated with favorable outcome. Area under the curve evaluated predictors of favorable clinical outcome.

RESULTS: Compared with the SPAN-100-negative group, the SPAN-100-positive group (55/273; 20\%) demonstrated larger CBVs (<0.001), poorer collaterals $(P<.001)$, and increased hemorrhagic transformation rates $(56.0 \%$ versus $36 \%, P=.02)$ despite earlier time to rtPA $(P=$ .03). Favorable outcome was less common among patients with SPAN-100-positive compared with SPAN-100-negative (10.9\% versus $42.2 \%$; $P<.001)$. Multivariate regression revealed poorer outcome for SPAN-100-positive (OR $=0.17 ; 95 \% \mathrm{Cl}, 0.06-0.38 ; P=.001)$, clot burden score $(\mathrm{OR}=1.14 ; 95 \% \mathrm{Cl}, 1.05-1.25 ; P<.001)$, and CBV $(\mathrm{OR}=0.58 ; 95 \% \mathrm{Cl}, 0.46-0.72 ; P=.001)$. The addition of the clot burden score and CBV improved the predictive value of SPAN-100 alone for favorable outcome from $60 \%$ to $68 \%$ and $74 \%$, respectively.

CONCLUSIONS: SPAN-100-positivity predicts a lower likelihood of favorable outcome and increased hemorrhagic transformation. CBV and clot burden score contribute to poorer outcomes among high-risk patients and improve stroke-outcome prediction.

ABBREVIATIONS: $\mathrm{AUC}=$ area under curve; $\mathrm{CBS}=$ clot burden score; SPAN-100 $=$ Stroke Prognostication Using Age and NIHSS

S everal scores have been designed to prognosticate clinical outcomes in acute ischemic stroke and assess potential risks of intravenous thrombolysis. ${ }^{1}$ Age and stroke severity measured by the National Institutes of Health Stroke Scale are among major independent prognostic factors for determining stroke outcome. $^{2,3}$ Stroke Prognostication Using Age and NIHSS (SPAN-

Received April 6, 2014; accepted after revision October 16.

From the Division of Neuroradiology (P.K., S.S., R.A.), Department of Medical Imag ing, University of Toronto and Sunnybrook Health Sciences Centre, Toronto, Ontario, Canada; Stroke Outcome Reach Center (G.S.), Division of Neurology, Department of Medicine, St. Michael's Hospital, University of Toronto, Ontario, Canada; Department of Neurosciences (B.O.), Medical University of South Carolina, Charleston, South Carolina; and Biostatistician (L.Z.), Sunnybrook Health Sciences Center, Toronto, Ontario, Canada.

P. Krishnan and G. Saposnik contributed equally to the article.

Paper previously presented at: American Society of Neuroradiology Annual Meet ing and the Foundation of the ASNR Symposium, May 17-22, 2014; Montreal, Quebec, Canada.

Please address correspondence to Richard I. Aviv, MD, FRCR, Sunnybrook Health Sciences Centre, 2075 Bayview Ave, Toronto, Ontario, Canada M4N 3M5; e-mail: richard.aviv@sunnybrook.ca

http://dx.doi.org/10.3174/ajnr.A4195
100) was conceived by combining age in years and stroke severity measured by the NIHSS ${ }^{4}$ and applying the combination to predict clinical outcome and risk of intracerebral hemorrhage. With individuals older than 80 years of age constituting a significant proportion of hospitalized patients with acute ischemic stroke, the relevance of the SPAN-100 is self-evident. ${ }^{5}$ Moreover, the elderly also have a higher risk of fatality and longer hospitalization, necessitating the consideration of the benefit-harm ratio preceding rtPA therapy. More interestingly, most stroke predictive scores use either clinical or imaging components, and though several exist, their utility in clinical practice is somewhat restricted. ${ }^{1}$ Multimodal imaging-selection strategies are evolving into a cornerstone for stroke management to best define target groups with salvageable tissue at risk. ${ }^{6-9}$ Apart from excluding hemorrhage and early ischemic changes, the presence and extent of ischemic core, intravascular clot burden, and extent of collaterals are critical elements assessed by imaging, dictating management and outcome in patients with stroke. ${ }^{10}$

The simplicity of SPAN-100, using readily accessible information including age and NIHSS, makes it attractive for practical 
use. Furthermore, imaging features accompanying SPAN-100positivity provide insight into pathophysiologic characteristics of patients evaluated with SPAN-100. We sought to externally validate SPAN-100, document multimodal CT parameters associated with SPAN-100 status, and assess their interaction with SPAN100 and clinical outcome.

\section{MATERIALS AND METHODS \\ Study Design and Patient Cohort}

A single-center retrospective study of patients presenting to a regional stroke center within 4.5 hours of anterior circulation stroke symptoms with a vessel occlusion, between October 2009 and December 2011, was performed. The institutional review board approved this study, and individual patient consent was obtained. Patients underwent clinical assessment by certified stroke neurologists and an acute CT-based stroke protocol, including CTA and CTP. Follow-up imaging included repeat CTA/CTP at 24 hours and 5- to 7-day CT or MR imaging. Presenting demographic data collected included age, sex, and cerebrovascular risk factors, including hypertension, diabetes, hypercholesterolemia, coronary artery disease, atrial fibrillation, and history of smoking and previous stroke. Baseline National Institutes of Health Stroke Scale (pre-NIHSS) and 3-month follow-up modified Rankin Scale scores were documented. Patients were not treated with intraarterial therapies because this option was not available when these patients underwent the CT studies.

Outcome Measures. The primary outcome was favorable clinical outcome defined as mRS $\leq 2$. Secondary outcomes included hemorrhagic transformation (by using the European Cooperative Acute Stroke Study definition), recanalization, and lack of NIHSS improvement between baseline and 24 hours (defined as $<3$ point NIHSS change). ${ }^{4}$ Patients were divided into 2 groups: SPAN-100-positive (age + NIHSS score of $>100$ ) and SPAN100-negative (age + NIHSS score of $<100$ ).

Image Analysis. Imaging was assessed by an experienced neuroradiologist. A comprehensive imaging review documented the presence of the hyperattenuated vessel sign, ASPECTS, early ischemic changes, clot burden score, and collateral score. CBV, CBF, and MTT volumes were measured planimetrically by using Medical Image Processing, Analysis, and Visualization, Version 4.4.1 (National Institutes of Health, Bethesda, Maryland; http:// mipav.cit.nih.gov). The threshold adopted for volumetric measurements of penumbra and infarct was internally validated. Penumbral tissue was identified by using a threshold of CBF of $>19$ $\mathrm{mL} / 100 \mathrm{~g} / \mathrm{min}$ and relative MTT of $<140 \%$, whereas infarct on the CBV map was defined by CBV of $<1.48 \mathrm{~mL} / 100 \mathrm{~g} .{ }^{11} \mathrm{CT}$ Perfusion software, Version 4 (GE Healthcare, Milwaukee, Wiscon$\sin$ ) was used to analyze data from the baseline CT perfusion study to calculate parametric maps of CBF, CBV, and MTT. A deconvolution of the arterial input curves by using the model of Johnson and Wilson was applied to calculate the parametric maps. ${ }^{12} \mathrm{~A}$ venous output function from the anterior cerebral artery and the superior sagittal sinus was obtained to correct for partial volume averaging of the arterial input curves. Functional CT perfusions maps were analyzed by using custom software (IDL, Version 6.1; RSI-Research Systems, Chapel Hill, North Carolina). All compo- nents of the analysis were performed blinded to the clinical information to reduce interpreter bias. Pixels with CBF values of $>100$ $\mathrm{mL} / 100 \mathrm{~g} / \mathrm{min}$ or $\mathrm{CBV}$ of $>8 \mathrm{~mL} / 100 \mathrm{~g}$ were excluded and were not used for calculating average $\mathrm{CBF}$ and $\mathrm{CBV}$ values for regions of interest. The time from symptom onset to scan, rtPA treatment, and hemorrhagic transformation on follow-up was noted for each patient.

Clot burden score (CBS) was used to quantify the burden of intracranial thrombus in the proximal intracranial circulation. The score allocates points on a scale of $0-10$ for contrast opacification of proximal intracranial vessels. Two points each were subtracted for the presence of thrombus in the supraclinoid ICA and proximal or distal M1, and 1 point each, for the infraclinoid ICA, $\mathrm{A} 1$, and each affected proximal M2 branch ( $\leq 2$ points). ${ }^{12}$ Collateral score was used to grade the extent of collateral vascular supply to the occluded MCA distribution on a scale of $0-3$. A score of zero denotes absent collateral supply; a score of 1 , collateral filling of $\leq 50 \%$; a score of $2,>50 \%$ but $<100 \%$; and a score of 3 , collateral supply to $100 \%$ of the occluded MCA distribution. ${ }^{12}$

\section{Scanning Protocol and Generation of Parametric Maps}

The CT stroke protocol was performed on a 64-section CT scanner (LightSpeed VCT; GE Healthcare) and included pre- and postcontrast CT head scans. The parameters used were as follows: $120 \mathrm{kV}$ (peak), $340 \mathrm{~mA}, 8 \times 5 \mathrm{~mm}$ collimation, $1 \mathrm{~s} /$ rotation, and table speed of $15 \mathrm{~mm} /$ rotation. Standard CTA from the aortic arch to the vertex was performed with the following parameters: $0.7-\mathrm{mL} / \mathrm{kg}$ iodinated contrast, maximum of $90 \mathrm{~mL}$ (iohexol; Omnipaque, $300 \mathrm{mg}$ iodine/mL; GE Healthcare), 5- to 10-second delay, $120 \mathrm{kVp}, 270 \mathrm{~mA}, 1$ s/rotation, 1.25-mm-thick sections, and table speed of $3.7 \mathrm{~mm} /$ rotation. CTA data comprised multiplanar 7-mm MIP reconstructions and 4-mm axial reformats on CTA source images. The biphasic CTP technique included a 45second initial scan reconstructed at 0.5 -second intervals, producing a series of 90 sequential images for each of the 8 sections, covering $4 \mathrm{~cm}$ from the basal ganglia to the lateral ventricles. This was followed by a second phase covering the same 8 sections, 15 seconds apart for 6 acquisitions for an additional 90 seconds as previously published. ${ }^{13}$

CTP scanning parameters used were the following: $80 \mathrm{kVp}$, $100 \mathrm{~mA}, 0.5-\mathrm{mL} / \mathrm{g}$ (maximum, 50-mL) iodinated contrast agent injected at $4 \mathrm{~mL} / \mathrm{s}$ with a 3 - to 5 -second delay.

\section{Statistical Analysis}

All analyses were conducted by SAS (Version 9.3 for Windows; SAS Institute, Cary, North Carolina). We compared demographic and clinical factors between patients with SPAN-100-positivity and -negativity. The $\chi^{2}$ test was used for categoric variables; the Wilcoxon rank sum test, for continuous variables with nonnormalized distribution; and the ANOVA, for those with normalized distribution. To search for the most significant clinical and imaging factors related to SPAN-100 status, we performed backward stepwise-selection logistic regression. Natural logtransformation was applied for normalization of variables when necessary. Comparison of demographic, imaging, and outcome factors was made for patients with SPAN-100-positivity and SPAN-100-negativity who did or did not receive rtPA, by using univariate logistic regression. To investigate the 
Table 1: Comparing demographics/clinical factors between patients with positive SPAN-100 and patients with negative SPAN-100

\begin{tabular}{|c|c|c|c|}
\hline & $\begin{array}{l}\text { SPAN-100-Negative } \\
\qquad(n=218)\end{array}$ & $\begin{array}{c}\text { SPAN-100-Positive } \\
(n=55)\end{array}$ & $P$ Value \\
\hline Age (yr) & $68.2 \pm 12.5$ & $85.5 \pm 5.07$ & $<.001$ \\
\hline NIHSS (median, IQR) & $13(7-18)$ & $21(17-24)$ & $<.001$ \\
\hline Male sex & $121(55.5 \%)$ & $22(40)$ & .04 \\
\hline SBP & 157.7 (139-172) & 156.04 (138-177) & .77 \\
\hline DBP & 84.9 (71-95) & $76.1(64-87)$ & .01 \\
\hline Glucose (admission) & $8.1(5.8-8.1)$ & $7.6(6.0-9.0)$ & .18 \\
\hline \multicolumn{4}{|l|}{ Risk factors } \\
\hline Hypertension & $127(58.26)$ & $45(81.8)$ & .001 \\
\hline Diabetes mellitus & 43 (19.72) & 10 (18.18) & .79 \\
\hline Hypercholesterolemia & $72(33.03)$ & $25(45.4)$ & .08 \\
\hline Coronary artery disease & $53(24.3)$ & $12(21.8)$ & .69 \\
\hline Atrial fibrillation & $64(29.36)$ & $19(34.5)$ & .45 \\
\hline Smoker & $44(20.1)$ & $6(10.9)$ & .11 \\
\hline Stroke & $1(0.46)$ & $1(1.82)$ & .29 \\
\hline Hyperdense sign & $114(52.53)$ & $35(63.64)$ & .13 \\
\hline ASPECTS (median) (IQR) & $7(6-9)$ & $7(5-8)$ & .39 \\
\hline Clot burden score & $6(4-9)$ & $6(5-9)$ & .29 \\
\hline Collateral score & $2(2-3)$ & $2(1-2)$ & $<.001$ \\
\hline CBV (median) (IQR) & $14.7(4.7-34.7)$ & $34.7(13.8-60.5)$ & $<.001$ \\
\hline $\mathrm{CBF}$ & $101.6(55.3-133.1)$ & $98.2(74.6-129.5)$ & .75 \\
\hline MTT & $104.5(58.5-133.4)$ & $98.4(74.7-130.5)$ & .69 \\
\hline \multicolumn{4}{|l|}{ Time and thrombolysis } \\
\hline rtPA dose & $63.0(54-73)$ & $63.0(54-72)$ & .75 \\
\hline Onset to CT & $104.0(80-148)$ & $108.0(75-127)$ & .62 \\
\hline Onset to rtPA & $161.7(147)$ & $143.0(131)$ & .03 \\
\hline \multicolumn{4}{|l|}{ Outcome } \\
\hline Recanalization & $119(55.35)$ & $33(61.1)$ & .44 \\
\hline mRS (at follow-up) & $3(1-4)$ & $5(4-6)$ & $<.001$ \\
\hline $\mathrm{mRS} \leq 2$ & $92(42.2)$ & $6(10.9)$ & $<.001$ \\
\hline NIHSS improves $>3$ in 24 hours & $101(46.3)$ & $27(49)$ & .71 \\
\hline Hemorrhagic transformation & $78(38.6)$ & $28(56.0)$ & .02 \\
\hline Hemorrhage infarct & 64 (29.4) & $23(41.8)$ & .07 \\
\hline Parenchymal hemorrhage & $26(11.9)$ & $9(16.4)$ & .37 \\
\hline
\end{tabular}

Note:- IQR indicates interquartile range; SBP, systolic blood pressure; DBP, diastolic blood pressure. and diastolic blood pressure $(P=.006)$. rtPA was given to $47 / 55(85.5 \%)$ of the SPAN-100-positive group and 172/218 (78.9\%) of the SPAN-100-negative group $(P=.28)$. The rtPA dose was comparable in both groups with a mean of $63 \mathrm{mg}(P=$ $.7)$, though patients with SPAN-100-positivity received rtPA earlier (143 minutes versus 161 minutes; $P=.03$ ).

\section{Differences in Imaging Parameters by SPAN-100 Status}

The collateral score was lower in patients with SPAN-100-positivity, consistent with worse collateral circulation $(P<$ .001). Baseline CBVs were higher in patients with SPAN-100-positivity $(P=.001)$ despite similar CBF/MTT volumes ( $P=.7$ and .6 , respectively), indicating comparable degrees of ischemia. No significant difference for median baseline ASPECTS ( $P=.39$ ), hyperattenuated MCA sign, clot burden score, or early ischemic change was observed. Stepwise multivariate logistic regression analysis revealed that hypertension $(\mathrm{OR}=3.1$; 95\% CI, $1.1-$ $7.07 ; P=.003)$, female sex $(\mathrm{OR}=0.47$; $95 \% \mathrm{CI}, 0.2-0.8 ; P=.02)$, and collateral score $(\mathrm{OR}=0.4 ; 95 \% \mathrm{CI}, 0.2-0.6$; $P<.001)$ were associated with SPAN100-positive status. Baseline CBV did not reach clinical significance. association between favorable outcome and demographic/ clinical factors, we performed a univariate logistic regression analysis as described above. Factors with $P<.10$ in univariate analysis were included in a backward stepwise logistic regression after adjusting for SPAN-100 status.

The additional benefit of significant clinical and radiologic factors over SPAN-100 as a null model for favorable outcome prediction was tested by using the Akaike information criterion (AIC $\left.=L_{\mathrm{RES}}+2 \times k\right)$. A lower Akaike information criterion indicates a better model fit, where $L_{\mathrm{RES}}$ represents the restricted maximized $-2 \times \log$-likelihood $(-2 \mathrm{~L})$ of the model, and $k$, the number of parameters in the model. The G2 likelihood ratio statistic is the difference between $-2 L$ of the fitted model (transformed threshold) and the reference model (nontransformed threshold). A 2-sided $P$ value was obtained from the G2 likelihood ratio $\chi^{2}$ test. Similarly, the area under the curve (AUC) was calculated for each model by using receiver operator characteristic curves and was compared with pair-wise comparison. A $P$ value $<$ .05 was considered significant.

\section{RESULTS}

Among 273 patients with acute ischemic stroke, 55 (20.1\%) were SPAN-100-positive (Table 1). Factors associated with SPAN-100positivity included female sex $(P=.02)$, hypertension $(P=.001)$,

\section{Primary and Secondary Outcome by SPAN-100 Status}

Favorable clinical outcome was less common in patients with SPAN-100-positivity ( $10.9 \%$ versus $42.2 \%, P<.001$ ). Any hemorrhage was more common in patients with SPAN-100-positivity (56\% versus $38.6 \%, P=.02$ ). Hemorrhagic transformation was the most common hemorrhagic infarction subtype (Table 1). Recanalization rate and lack of NIHSS improvement were similar $(P=.4$ and $P=.7)$. In contradistinction to patients with SPAN100 -negativity, no significant demographic imaging or outcome differences were present in patients with SPAN-100-positivity with or without rtPA treatment. Patients with SPAN-100-negativity treated with rtPA were more likely than non-rtPA-treated patients to present earlier $(P=.01)$, demonstrate the hyperattenuated sign $(P=.06)$, undergo hemorrhagic infarction $(P=$ $.05)$, recanalization $(P=.009)$, show neurologic improvement within 24 hours, and experience a good clinical outcome $(P=.06)$.

\section{Predictors of Outcome}

Multiple clinical and radiologic factors were associated with favorable clinical outcome on univariate analysis (Table 2). The multivariate logistic regression showed that SPAN-100-positivity $(\mathrm{OR}=0.17 ; 95 \% \mathrm{CI}, 0.06-0.38 ; P<.001)$ and larger $\mathrm{CBV}(\mathrm{OR}=$ $0.58 ; 95 \% \mathrm{CI}, 0.46-0.72 ; P<.001)$ were associated with a lower 
Table 2: Univariate logistic regression analysis of good clinical outcome ( $m R S \leq 2)$ on demographic and clinical factors and imaging parameters

\begin{tabular}{|c|c|c|c|c|}
\hline & $\begin{array}{c}\mathrm{mRS} \leq 2(n=98), \\
\text { Favorable } \\
\text { Outcome }\end{array}$ & $\begin{array}{c}\mathrm{mRS}>2(n=175) \\
\text { Poor } \\
\text { Outcome }\end{array}$ & $\begin{array}{c}P \\
\text { Value }\end{array}$ & OR $(95 \% \mathrm{Cl})$ \\
\hline Age (yr) & $67(57-78)$ & $76(66-83)$ & $<.001$ & $0.96(0.94-0.98)$ \\
\hline NIHSS pre-rtPA (median, IQR) & $17(12-21)$ & $9.0(4-15)$ & $<.001$ & $0.86(0.82-0.90)$ \\
\hline Female sex & $42(42.86)$ & $88(50.29)$ & .23 & $1.35(0.82-2.23)$ \\
\hline SBP & $151(138.5-173.5)$ & 155 (137-171.5) & .93 & $1.00(0.99-1.01)$ \\
\hline DBP & $81(72.5-92)$ & $80(70-90)$ & .26 & $1.01(0.99-1.02)$ \\
\hline Glucose (admission) $^{a}$ & $6.4(5.4-7.3)$ & $6.4(5.4-7.3)$ & .04 & $0.42(0.17-0.92)$ \\
\hline \multicolumn{5}{|l|}{ Risk factors } \\
\hline Hypertension & $54(55.10)$ & 118 (67.43\%) & .04 & $0.59(0.36-0.99)$ \\
\hline Diabetes mellitus & $15(15$ & $38(2$ & .20 & $0.65(0.33-1.24)$ \\
\hline Hypercholesterolemia & $29(29.59)$ & $68(38.86)$ & .12 & $0.66(0.39-1.12)$ \\
\hline Coronary artery disease & $23(24.3)$ & $42(24)$ & .92 & $0.97(0.54-1.73)$ \\
\hline Atrial fibrillation & $29(29$ & $54(30.86)$ & .82 & $0.94(0.55-1.61)$ \\
\hline Smoker & $22(22.45)$ & $28(16)$ & .18 & $1.52(0.81-2.83)$ \\
\hline Stroke & $1(1.02)$ & $1(0.57)$ & .68 & $1.79(0.07-45.66)$ \\
\hline Hyperdense sign & $48(48.98 \%)$ & $101(58.05)$ & .14 & $0.69(0.42-1.14)$ \\
\hline EIC & $84(85.91)$ & $158(90.29)$ & .25 & $0.65(0.30-1.39)$ \\
\hline ASPECTS (median, IQR) & $8(6-9)$ & $7(5-8)$ & .001 & $1.24(1.09-1.42)$ \\
\hline Clot burden score & $7(6-9)$ & $6(4-9)$ & .002 & $1.14(1.05-1.25)$ \\
\hline Collateral score & $2(2-3)$ & $2(1-3)$ & .02 & $1.46(1.06-2.04)$ \\
\hline CBV (median, IQR) ${ }^{\mathrm{a}}$ & $8.77(2.5-24.09)$ & $25.14(9.1-49.60)$ & $<.001$ & $0.58(0.46-0.72)$ \\
\hline $\mathrm{CBF}$ & $88.12(44.7-122.1)$ & $104.17(74.3-143.6)$ & $<.001$ & $0.99(0.99-1.00)$ \\
\hline MTT & $87.96(43.2-125.9)$ & $105.4(77.5-138.8)$ & $<.001$ & $0.99(0.99-1.00)$ \\
\hline \multicolumn{5}{|l|}{ Time and thrombolysis } \\
\hline rtPA given (No. of the patients) & 80 (81.63\%) & 139 (79.43\%) & .66 & $1.15(0.62-2.20)$ \\
\hline rtPA dose $(\mathrm{mg})$ & $64.0(50-73)$ & $62.0(54-72)$ & .94 & $1.00(0.98-1.02)$ \\
\hline Onset to $\mathrm{CT}$ (min) (median, IQR) & $102.0(74-151)$ & $105.0(80.5-141.5)$ & .73 & $1.08(0.68-1.72)$ \\
\hline Onset to rtPA (min) (median IQR) & 145 (120-179) & $146(126-175)$ & .70 & $1.00(1.00-1.01)$ \\
\hline \multicolumn{5}{|l|}{ Outcome } \\
\hline Recanalization & 119 (55.35\%) & $33(61.1 \%)$ & $<.001$ & $3.58(2.09-6.30)$ \\
\hline Hemorrhagic transformation & $28(30.43 \%)$ & $78(48.75 \%)$ & .004 & $0.46(0.26-0.78)$ \\
\hline NIHSS improves $>3$ in 24 hours & $101(46.3 \%)$ & $27(49 \%)$ & .20 & $1.38(0.84-2.27)$ \\
\hline SPAN-100-positive & $6(6.12 \%)$ & $49(28 \%)$ & $<.001$ & $0.17(0.06-0.38)$ \\
\hline
\end{tabular}

${ }^{a}$ Natural log-transformation was applied for normalizing the distribution.

Table 3: Prediction of favorable clinical outcome and hemorrhagic transformation

\begin{tabular}{|c|c|c|c|c|c|c|c|}
\hline & AIC & $\begin{array}{c}-2 \\
\text { (Log-Likelihood) }\end{array}$ & $R^{2 \mathrm{a}}$ & AUC & OR & $95 \% \mathrm{Cl}$ & $\begin{array}{c}P \\
\text { Value }\end{array}$ \\
\hline \multicolumn{8}{|l|}{ Favorable clinical outcome } \\
\hline Model of SPAN-100 only & 316.64 & 312.64 & 0.054 & 0.599 & & $0.56-0.64$ & \\
\hline SPAN-100 & & & & & 0.2 & $0.1-0.4$ & \\
\hline Model of SPAN-100, CBS & 307.42 & 301.42 & 0.088 & 0.676 & & $0.61-0.74$ & .004 \\
\hline SPAN-100 & & & & & 0.2 & $0.1-0.3$ & \\
\hline CBS & & & & & 1.2 & $1.1-1.3$ & \\
\hline Model of SPAN-100, CBS, CBV & 294.09 & 286.09 & 0.135 & 0.742 & & $0.68-0.80$ & $<.001$ \\
\hline SPAN-100 & & & & & 0.2 & $0.1-0.5$ & \\
\hline CBS & & & & & 1.1 & $1.0-1.2$ & \\
\hline CBV & & & & & 0.6 & $0.5-0.8$ & \\
\hline \multicolumn{8}{|l|}{ Hemorrhagic transformation } \\
\hline Model of SPAN-100 only & 342.06 & 338.06 & 0.019 & 0.55 & & $0.49-0.60$ & \\
\hline SPAN-100 & & & & & 2.0 & $1.1-3.8$ & \\
\hline Model of SPAN-100, CBS & 339.39 & 333.392 & 0.037 & 0.62 & & $0.54 .5-0.69$ & .02 \\
\hline SPAN-100 & & & & & 2.2 & $1.2-4.1$ & \\
\hline CBS & & & & & 0.9 & $0.8-0.9$ & \\
\hline
\end{tabular}

Note:-AIC indicates Akaike information criterion.

${ }^{a} R^{2}$ is the proportion of variability in a dataset that is accounted for by the statistical model.
Note:-EIC indicates early ischemic changes.

$60 \%$, increasing significantly to $68 \%$ and $74 \%$ with the addition of the clot burden score and CBV, respectively (Table 3). Univariate logistic regression for any hemorrhage showed significance for NIHSS $(P<.001)$, hyperattenuated sign $(P=.001)$, ASPECTS $(P=.022)$, collateral score $(P=.020)$, CBV $(P<.001)$, and SPAN-100 $(P=.027)$. Stepwise logistic regression of SPAN-100, collateral score, CBV, and clot burden score demonstrated significant associations for SPAN-100 $(\mathrm{OR}=2.17$; 95\% CI, $1.16-$ 4.14; $P=.001)$ and clot burden score $(\mathrm{OR}=0.91 ; 95 \% \mathrm{CI}, 0.84-0.99 ; P=$ $.03)$ with hemorrhagic transformation. The AUC for hemorrhagic transformation by using SPAN-100 alone was 55\%, increasing significantly to $62 \%$ with the addition of clot burden score (Table 3 ).

\section{DISCUSSION}

Early prognostication of ischemic stroke outcome is a critical component of stroke management. SPAN-100, combining age and NIHSS score, is a recently proposed simple and practical tool to estimate the clinical response and risk of hemorrhagic complications after thrombolysis. ${ }^{4}$ Although other clinical risk scores ${ }^{1}$ may have a better power to prognosticate stroke, the SPAN-100 index is a practical tool that may help determine patients who are more likely to achieve a good or poor outcome. The combination of a simple clinical tool with imaging parameters may help stratify patients according to their risk for receiving thrombolytic or endovascular therapy for acute stroke. We evaluated the role of imaging parameters added to the SPAN-100 score to estimate clinical outcomes. We confirmed that patients with SPAN-100-positivity were less likely to be independent irrespective of rtPA treatment, while carrying a higher risk of hemorrhagic complications. Notably, worse outcomes occurred despite earlier time to rtPA therapy. Patients with SPAN-100-positivity had lower collateral circulation and larger baseline

likelihood of a favorable outcome, whereas a higher clot burden score $(\mathrm{OR}=1.14 ; 95 \% \mathrm{CI}, 1.05-1.25 ; P=.003)$ was associated with a greater chance of a favorable outcome. No interaction was found between SPAN-100 and CBV $(P=.53)$ or between SPAN100 and clot burden score $(P=.98)$, respectively. The AUC for favorable clinical outcome by using SPAN-100 alone was
CBVs. Extending prior studies, we explored the interaction between the SPAN-100 index and additional radiologic factors and assessed their additional predictive value over SPAN-100 status for clinical outcome. Both the clot burden score (reflecting the burden of intraluminal thrombus) and CBV (reflecting infarct core) remained significant contributors to clinical out- 

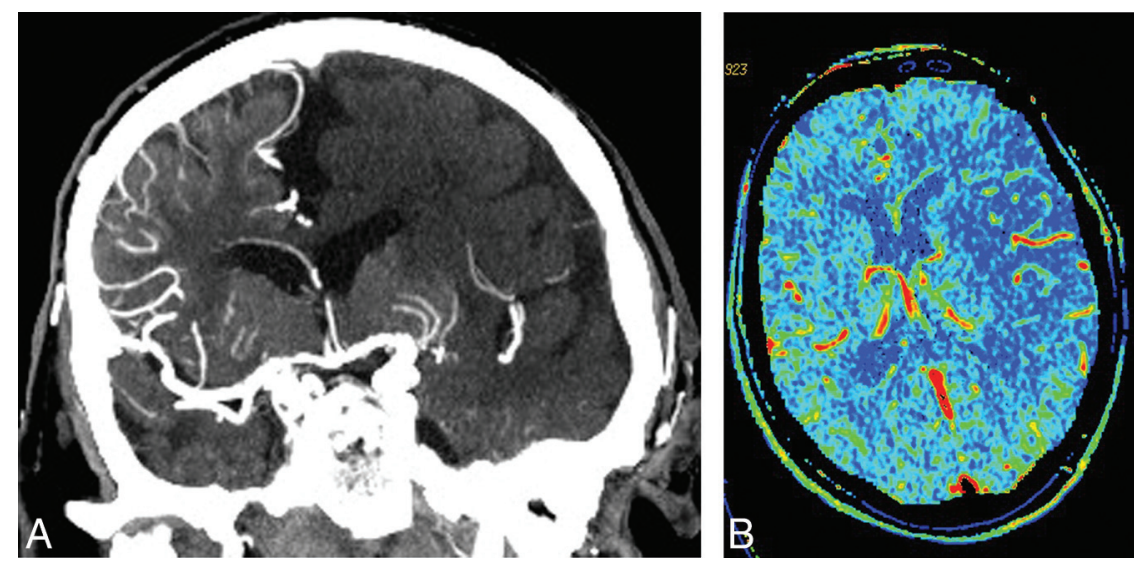

FIG 1. Coronal CTA MIP image and CBV map in a patient with SPAN-100-positivity at presentation. Coronal CTA MIP image $(A)$ in this SPAN-100-positive patient with acute right-sided hemiparesis and subsequent unfavorable outcome demonstrates abrupt occlusion of the main stem left MCA with a collateral score of zero. The corresponding CBV map $(B)$ demonstrates a large CBV deficit.
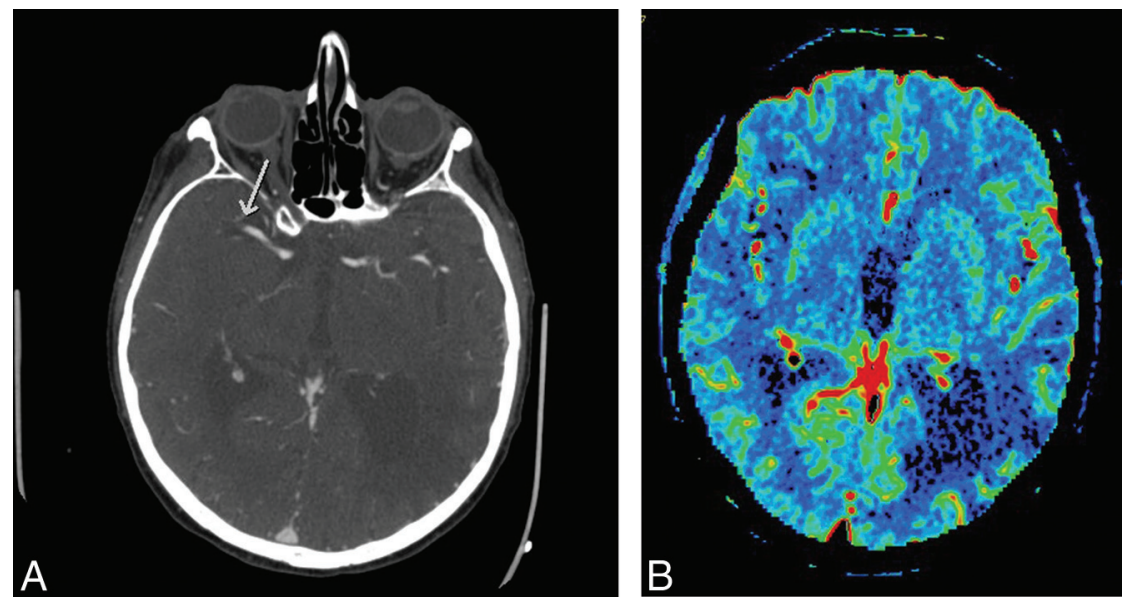

FIG 2. Axial CTA MIP image and CBV map in a patient with SPAN-100-positivity with favorable outcome. Axial CTA MIP $(A)$ in this SPAN-100-positive patient with left-sided acute stroke and favorable outcome shows abrupt occlusion of the distal main stem right MCA. The extent of clot burden is low and underscores the utility of imaging in prognostication. The CBV map $(B)$ shows a cortical-subcortical insular/subinsular defect in the right MCA distribution, with relative sparing of the basal ganglia. Chronic infarction is incidentally seen in the left parietal lobe. ume and hemorrhage as previously described. ${ }^{19,20}$ Despite CBV differences in SPAN-100 subgroups on univariate analysis, no significant baseline difference of ASPECTS was seen. This apparent disparity likely reflects the relatively coarse sensitivity of ASPECTS for lesion volume within the M1-6 or cortical regions compared with the significant impact of even small basal ganglia lesions on ASPECTS. Heavy weighting of ASPECTS to the basal ganglia may, in part, contribute to its modest sensitivity for outcome prediction. $^{21,22}$

Although reduced collateral flow was also associated with unfavorable outcome on univariate analysis, CBV and the clot burden score remained dominant in the multivariate analysis in addition to SPAN-100 status. In the multivariate analysis, the imaging factors of SPAN-100 status, CBV, and clot burden scores were significantly associated with the mRS outcome (Fig 2). The lack of interaction between SPAN-100 status and clot burden score or between CBV and SPAN-100 status indicated that the probability difference on the mRS good outcome between SPAN-100-positivity and -negativity was similar with clot burden score changes or with $\mathrm{CBV}$ changes. For instance, in patients with a clot burden score of 6 , the proportion of good mRS outcome was $6 \%$ for patients with positive SPAN-100 and 49\% for patients with negative SPAN-100 (the difference was $43 \%$ ). In patients with a clot burden score of 9 , the difference in the proportion of good mRS outcome was $33 \%$ versus $71 \%$ for those with positive come prediction and improved the prediction of the probability of achieving a favorable outcome at 3 months. Similarly the SPAN-100 index and clot burden score predicted hemorrhagic transformation.

In patients with SPAN-100-positivity, reduced collateral flow contributed to larger baseline $\mathrm{CBV}$ s manifest by its dominance within a multivariate analysis of SPAN-100 status and the loss of CBV significance. Collateral score reduction in patients with SPAN-100positivity is supported by prior reports of diminishing functional collateral compensatory capacity with age. ${ }^{14}$ Similarly, the CTA collateral profile is strongly associated with baseline infarct volume and long-term outcome in acute ischemic stroke. ${ }^{15-17}$ Poor collateral flow and larger $\mathrm{CBV}$, in part, explain the worse outcome in patients with SPAN-100-positivity despite an earlier time to rtPA (Fig 1). Increased intracranial hemorrhage in patients with SPAN-100-positivity may also, in part, be attributed to poorer collaterals ${ }^{18}$ and larger baseline CBVs, with a clear association between baseline infarct vol- or negative SPAN-100 (the difference was 38\%). These nonsignificant interaction terms might be due to the limited sample size in patients from the SPAN-100-positive group. Indeed, similar to a prior study, the AUC of SPAN-100 status alone was $60 \% .{ }^{23}$ The addition of $\mathrm{CBV}$ and clot burden score, however, increased predictions to $74 \%$. A systematic review by Schiemanck et $\mathrm{al}^{24}$ and several other studies ${ }^{7,10,25}$ corroborated the importance of lesion volume and neurologic deficit assessed by the NIHSS score for clinical-outcome determination. Clot location and volume were both previously shown to be important independent prognostic factors of outcome. ${ }^{10,12,26}$ Similarly, larger clot burden is associated with larger baseline infarct volumes, poorer clinical outcome, and risk of hemorrhage. ${ }^{26,27}$

Limitations of the present study include a retrospective data analysis with a modest sample size. CBV, though improving prognostication for outcome, is difficult to measure in real-time and complicates the purposeful simplicity of the SPAN-100 index as a 
quick clinical prognostic tool. Whether rapid estimations of CBV, for example by $\mathrm{ABC} / 2$ (a commonly used method for volume calculation), provide benefit similar to that of a planimetric approach remains uncertain. The limited spatial resolution of CT perfusion may also underestimate complete CBV measurement. This issue is easily addressed with widely available table-toggle techniques ${ }^{28,29}$ or 320 -section scanners capable of whole-head imaging. Furthermore, the small sample of patients with SPAN100-positivity is a limitation of this study and could have partly contributed to the lack of a significant difference in outcomes in this group. Finally, the accuracy of CTP CBV for DWI core assessment has recently been questioned, ${ }^{30}$ though in our experience, this was largely mitigated by protocols that capture the full timeattenuation curve, thereby avoiding CBV underestimation.

Most of the predictive scores for outcome in acute stroke are inclusive of age and stroke severity scale (NIHSS), and their predictive power is moderate. Hence, consideration for rtPA treatment is currently based on clinical judgment, and clinical scores are used as an adjunct. Because the SPAN-100 index is among the more simplified prognostic scores for stroke outcome with core prognostic determinants of age and NIHSS, it would be reasonable to suggest that imaging parameters should be an integral part of the future stroke-outcome prediction scores with a need to customize for individual patients with a greater degree of precision. Our study re-emphasizes the need to incorporate imaging parameters (eg, CBV, collaterals, and clot burden scores) to provide additional predictive power.

The practical use of clinical prediction scores at present is limited in decision-making paradigms and is essentially complementary to clinical assessment. Future trials and larger retrospective studies inclusive of imaging parameters are needed to design comprehensive clinical scores with the potential to triage patients and tailor treatment options.

\section{CONCLUSIONS}

Imaging parameters improve outcome estimation in stroke prognostication when added to the clinical risk score (SPAN-100 index). Reduced collateral flow, higher clot burden, and larger cerebral blood volume deficits offer insight into the most relevant pathophysiologic parameters explaining poorer clinical outcomes among patients with SPAN-100-positivity.

The addition of imaging parameters to the SPAN-100 index improves the predictive power of stroke prognostication (ie, the prediction of favorable outcome and the risk of subsequent hemorrhage). The inherent simplicity of the SPAN-100 index and additional imaging parameters renders easy translation of this prediction score for practical use in routine clinical decision-making.

The routinely performed imaging assessment for acute stroke (multimodal CT and the parameters CBV, clot burden score, and collateral scores) could potentially add meaningful value to a wellestablished simplified clinical score (SPAN-100 index) for stroke prognostication.

Disclosures: Gustavo Saposnik—UNRELATED: Grants/Grants Pending: Dr Saposnik is supported by the Distinguished Clinician Scientist Award from the Heart and Stroke Foundation of Canada.

\section{REFERENCES}

1. Rabinstein A. Rundek T. Prediction of outcome after ischemic stroke: the value of clinical scores. Neurology 2013;80:15-16

2. Weimar C, Konig IR, Kraywinkel K, et al. Age and National Institutes of Health Stroke Scale Score within 6 hours after onset are accurate predictors of outcome after cerebral ischemia: development and external validation of prognostic models. Stroke 2004;35:158-62

3. König I, Ziegler A, Bluhmki E, et al. Predicting long-term outcome after acute ischemic stroke: a simple index works in patients from controlled clinical trials. Stroke 2008;39:1821-26

4. Saposnik G, Guzik A, Reeves M, et al. SPAN 100: a practical method of stroke prognostication combining age and NIHSS after tPA. Neurology 2013;80:21-28

5. Saposnik G, Cote R, Phillips S, et al. Stroke Outcome Research Canada (SORCan) Working Group: variables associated with 7-day, 30day, and 1-year fatality after ischemic stroke. Stroke 2008;39: 2522-30

6. Obach V, Oleaga L, Urra X, et al. Multimodal CT-assisted thrombolysis in patients with acute stroke: a cohort study. Stroke 2011;42: 1129-31

7. Lev MH, Segal AZ, Farkas J, et al. Utility of perfusion-weighted CT imaging in acute middle cerebral artery stroke treated with intraarterial thrombolysis: prediction of final infarct volume and clinical outcome. Stroke 2001;32:2021-18

8. Campbell BC, Weir L, Desmond PM, et al. CT perfusion improves diagnostic accuracy and confidence in acute ischaemic stroke. J Neurol Neurosurg Psychiatry 2013;84:613-18

9. Hopyan J, Ciarallo A, Dowlatshahi D, et al. Certainty of stroke diagnosis: incremental benefit with CT perfusion over noncontrast CT and CT angiography. Radiology 2010;255:142-53

10. Demchuk A, Menon B, Goyal M. Imaging based selection in acute ischemic stroke trials: a quest for imaging sweet spots. Ann N Y Acad Sci 2012;1268:63-71

11. Eilaghi A, d'Esterre CD, Lee TY, et al. Toward patient-tailored perfusion thresholds for prediction of stroke outcome. AJNR Am J Neuroradiol 2014;35:472-77

12. Tan IY, Demchuk AM, Hopyan J, et al. CT angiography clot burden score and collateral score: correlation with clinical and radiologic outcomes in acute middle cerebral artery infarct. AJNR Am J Neuroradiol 2009;30:525-31

13. Aviv RI, d'Esterre CD, Murphy BD, et al. Hemorrhagic transformation of ischemic stroke: prediction with CT perfusion. Radiology 2009;250:867-77

14. Brozici M, van der Zwan A, Hillen B. Anatomy and functionality of leptomeningeal anastomoses: a review. Stroke 2003;34:2750-62

15. Souza L, Yoo A, Chaudhry Z, et al. Malignant CTA collateral profile is highly specific for large admission DWI infarct core and poor outcome in acute stroke. AJNR Am J Neuroradiol 2012;33:1331-36

16. Lima FO, Furie KL, Silva GS, et al. The pattern of leptomeningeal collaterals on CT angiography is a strong predictor of long-term functional outcome in stroke patients with large vessel intracranial occlusion. Stroke 2010;41:2316-22

17. Liebeskind D. Collateral perfusion: time for novel paradigms in cerebral ischemia. Int J Stroke 2012;7:309-10

18. Bang O, Saver J, Kim S, et al. Collateral flow averts hemorrhagic transformation after endovascular therapy for acute ischemic stroke. Stroke 2011;42:2235-39

19. Bhatt A, Vora A, Thomas A, et al. Lower pretreatment cerebral blood volume affects hemorrhagic risks after intra-arterial revascularization in acute stroke. Neurosurgery 2008;63:874-78; discussion 878-79

20. Jain A, Jain M, Kanthala A, et al. Association of CT perfusion parameters with hemorrhagic transformation in acute ischemic stroke. AJNR Am J Neuroradiol 2013;34:1895-900

21. Dzialowski I, Hill MD, Coutts SB, et al. Extent of early ischemic changes on computed tomography (CT) before thrombolysis: prognostic value of the Alberta Stroke Program Early CT Score in ECASS II. Stroke 2006;37:973-78

AJNR Am J Neuroradiol 36:646-52 Apr 2015 www.ajnr.org 651 
22. Menon BK, Puetz V, Kochar P, et al. ASPECTS and other neuroimaging scores in the triage and prediction of outcome in acute stroke patients. Neuroimaging Clin N Am 2011;21:407-23, xii

23. Vogt $G$, Laage $R$, Shuaib A, et al. Initial lesion volume is an independent predictor of clinical stroke outcome at day 90: an analysis of the Virtual International Stroke Trials Archive (VISTA) database. Stroke 2012;43:1266-72

24. Schiemanck SK, Kwakkel G, Post MW, et al. Predictive value of ischemic lesion volume assessed with magnetic resonance imaging for neurological deficits and functional outcome post stroke: a critical review of the literature. Neurorehabil Neural Repair 2006;20:492-502

25. Murphy A, Symons SP, Hopyan J, et al. Factors influencing clinically meaningful recanalization after IV-rtPA in acute ischemic stroke. AJNR Am J Neuroradiol 2013;34:146-52

26. Sillanpää N, Saarinen JT, Rusanen H, et al. Location of the clot and outcome of perfusion defects in acute anterior circulation stroke treated with intravenous thrombolysis. AJNR Am J Neuroradiol 2013;34:100-06

27. Puetz V, Dzialowski I, Hill MD, et al. Calgary CTA Study Group: intracranial thrombus extent predicts clinical outcome, final infarct size and hemorrhagic transformation in ischemic stroke: the clot burden score. Int J Stroke 2008;3:230-36

28. Roberts HC, Roberts TP, Smith WS, et al. Multisection dynamic CT perfusion for acute cerebral ischemia: the "toggling-table" technique. AJNR Am J Neuroradiol 2001;22:1077-80

29. Youn SW, Kim JH, Weon YC, et al. Perfusion CT of the brain using 40-mm-wide detector and toggling table technique for initial imaging of acute stroke. AJR Am J Roentgenol 2008;191:W120-26

30. Lev MH. Acute stroke imaging: what is sufficient for triage to endovascular therapies? AJNR Am J Neuroradiol 2012;33:790-92 